QRS interval $(<130 \mathrm{~ms})$ also have mechanical dyssynchrony and might, therefore, benefit from CRT.

The Cardiac Resynchronization Therapy in Patients with Heart Failure and Narrow QRS (RethinQ) study randomized 172 patients with a standard indication for an implantable cardioverter-defibrillator, a QRS interval of $<130 \mathrm{~ms}$ and evidence of mechanical dyssynchrony to either CRT or no CRT (controls). All individuals underwent successful implantation of a CRT device (the CRT feature was, however, turned off in the control patients) and completed a baseline evaluation at 14 days.

At 6 months, similar proportions of patients in the CRT and control groups showed an increase in peak oxygen consumption from baseline of at least $1.0 \mathrm{ml} / \mathrm{kg}$ body weight per min during cardiopulmonary exercise testing ( $46 \%$ and $41 \%$, respectively). In addition, no significant differences were found between the two groups with regard to quality-of-life score, results of 6 min walk test, or echocardiographic measures. A greater proportion of patients in the CRT group did, however, show improvement in NYHA class (54\% vs $29 \% ; P=0.006)$. Prespecified subgroup analyses showed that, in comparison with no intervention, CRT increased peak oxygen consumption in patients with a QRS interval of $\geq 120 \mathrm{~ms}(P=0.02)$ but had little effect in those with a QRS interval of $<120 \mathrm{~ms}(P=0.45)$.

On the basis of these results, the authors conclude that patients with heart failure and narrow QRS intervals do not benefit from CRT.

Original article Beshai JF et al. (2007) Cardiacresynchronization therapy in heart failure with narrow QRS complexes. N Engl J Med 357: 2461-2471

\section{Blunted heart rate dip while sleeping may aid prediction of mortality}

While evidence exists regarding the negative effects of high blood pressure on mortality, the correlation between heart rate and mortality has not been heavily investigated. Ben-Dov et al. report that lack of a reduction in heart rate from awake to nocturnal levels is independently associated with increased mortality.

The investigators analyzed information from a service database for the period 1991-2005. Heart rate data from $24 \mathrm{~h}$ ambulatory blood pressure monitoring were available for a total of 3,957 patients.

In Cox proportional hazard analysis adjusted for age, sex, BMI, treated diabetes, and treated hypertension, the magnitude of the dip between awake and sleeping heart rates was a strong predictor of mortality, independent of systolic blood pressure dip $(P<0.001$ trend for deciles of heart rate dip) and had greater predictive power than awake and sleeping heart rates. Female sex, a high BMI, older age and being treated for hypertension or diabetes were all factors associated with reduced $(<10 \%)$ heart rate dip.

In addition, when blood pressure dipping was taken into account together with the heart rate dip, it was shown that patients who lacked a $10 \%$ or more reduction in both blood pressure and heart rate had the highest mortality $(P=0.002)$.

The authors conclude that ambulatory heart rate adds prognostic information beyond other currently used ambulatory blood pressure measurements, and that an elevated sleeping heart rate-resulting in a smaller dip from the awake heart rate-may be of particular value in predicting mortality.

Original article Ben-Dov IZ et al. (2007) Blunted heart rate dip during sleep and all-cause mortality. Arch Intern Med 167: $2116-2121$

\section{Study shows an association between obesity and abdominal aortic aneurysm}

A paper has reported that several measures of obesity are independently associated with abdominal aortic aneurysm (AAA) in men. Previous studies have demonstrated an association between obesity and occlusive arterial disease, but this one by Golledge et al. is reportedly the first to demonstrate an association between obesity and AAA.

In this population-based study, the investigators used ultrasonography to screen 12,203 men, aged 65-83 years, for AAA. Obesity (defined by AHA criteria as waist circumference $\geq 102 \mathrm{~cm}$ ) was present in $39 \%$ of the study population. An AAA $\geq 30 \mathrm{~mm}$ was identified in $875(7.2 \%)$ members of the study population, $386(44 \%)$ of whom were obese (odds ratio [OR] 1.28, 95\% $\mathrm{Cl} 1.11-1.47)$. Following adjustment for other known risk factors, waist 